\title{
АППРОКСИМАЦИЯ ОБЛИКА КРИСТАЛЛА: ПЕРВЫЕ РЕЗУЛЬТАТЫ ДЛЯ ДВУМЕРНОГО СЛУЧАЯ
}

Степенщиков Д.Г.

Геологический институт КНЦ РАН, Апатиты, stepen@geoksc.apatity.ru

\section{Аннотация}

В статье представлены первые наработки по количественной оценке облика искажённого кристалла. В двумерном случае кристалл моделируется как многоугольник, а его облик аппроксимируется эллипсом. Отношение длин малой и большой полуосей эллипса предлагается в качестве числовой оценки облика искажённого двумерного кристалла.

Ранее в [1-3] нами было предложено обратиться к облику кристалла как к одной из наиболее простых его морфологических характеристик. При этом было отмечено, что понятие «облик», видимо, в силу своей простоты, допускает неоднозначное толкование [3]. При переходе от качественных понятий «вытянутость», «уплощённость», «изометричность» к количественным, необходимо чётко указать метод оценки облика кристалла. Эта точность важна, например, для исследования зависимости облика кристалла от степени его искажения.

Напомним идею нашего подхода. Искажённый кристалл заменяется эллипсоидом, отношения полуосей которого задают две координаты в единичном квадрате. Таким образом, облик кристалла задаётся всего одной точкой (рис. 1). При сдвигах граней кристалла вдоль своих нормалей (рост или растворение грани), облик меняется непрерывным образом, формируя некоторое связное множество точек в пределах единичного квадрата.
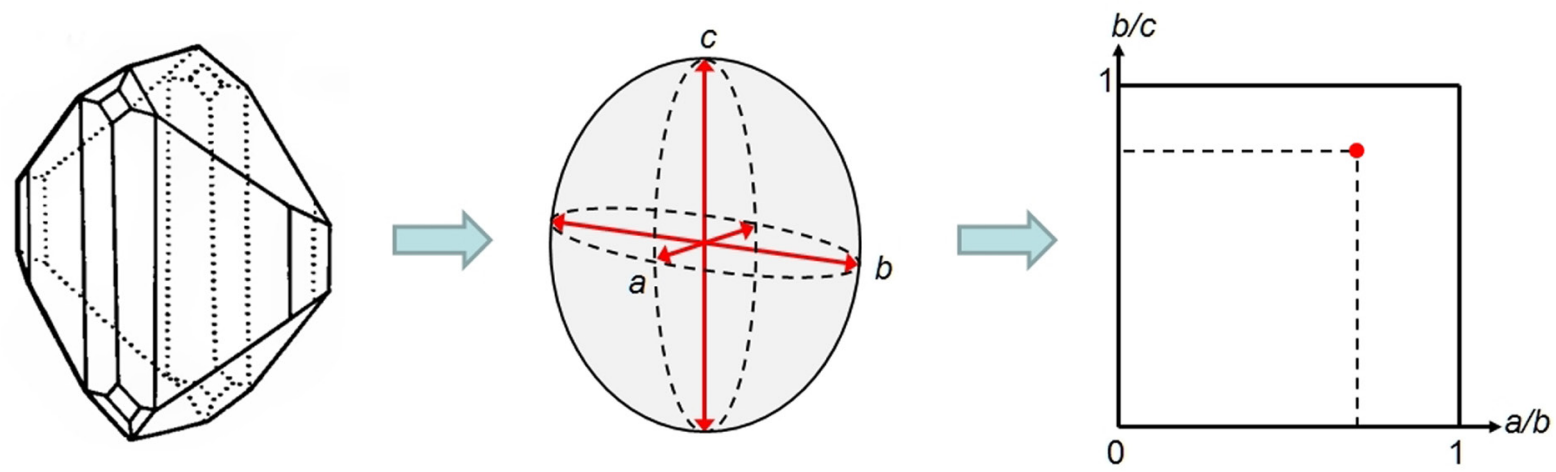

Рис. 1. Отображение облика кристалла в единичном квадрате.

Размеры и расположение эллипсоида, аппроксимирующего искажённый кристалл, подбираются так, чтобы обеспечить минимум объёма их непересекающихся частей (рис. 2). Последнее требование представляет особую сложность, так как ставит задачей подбор 9 параметров: координаты центра эллипсоида (3 числа), его ориентировку в пространстве (3) и длины его полуосей (3). 
В данной работе мы рассматриваем более простой двумерный случай. Кристаллы при этом имеют вид многоугольников и аппроксимируются эллипсами. Число неизвестных величин сокращается до 5: координаты центра эллипса (2), ориентировка эллипса на плоскости (1) и длины его полуосей (2). Данное понижение размерности, помимо упрощения, имеет и практический смысл как метод для описания морфологии плоских кристаллов [4].

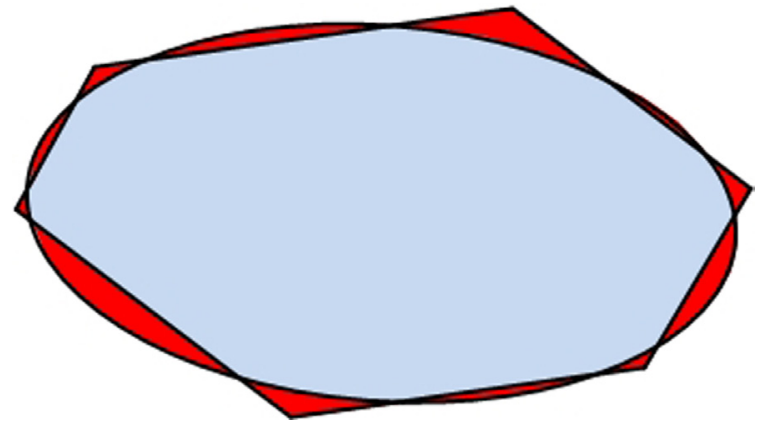

Рис. 2. Схема кристалла и аппроксимирующего его эллипсоида (непересекающиеся части выделены красным).

Для сокращения рутинного перебора возможных значений всех пяти параметров, нами было использовано несколько интуитивных допущений, которые, как показали последующие результаты, вполне корректны. Во-первых, за центр эллипса принимается барицентр (геометрический центр) многоугольника. Во-вторых, диапазон изменения обеих полуосей эллипса ограничивается сверху максимальным расстоянием от барицентра до вершины многоугольника (а снизу нулем). Такое равноправие полуосей, в свою очередь, позволяет сократить диапазон ориентировки (поворота) эллипса относительно многоугольника до $90^{\circ}$. В-третьих, предполагается, что при выбранной ориентировке эллипса и многоугольника зависимость суммарной площади их непересекающихся частей $S$ представляет собой непрерывную функцию $S(a, b)$ двух переменных (длин полуосей $a$ и $b$ ) с единственным минимумом $S_{\min }$. Это позволяет избежать прямого перебора и находить оптимальные значения полуосей эллипса, например, методом спуска. И, в-четвёртых, зависимость минимального значения $S_{\min }$ от угла поворота $\alpha$ предполагается непрерывной функцией $S_{\text {min }}(\alpha)$ с единственным минимумом. Все эти эвристики представляют отдельный теорети-
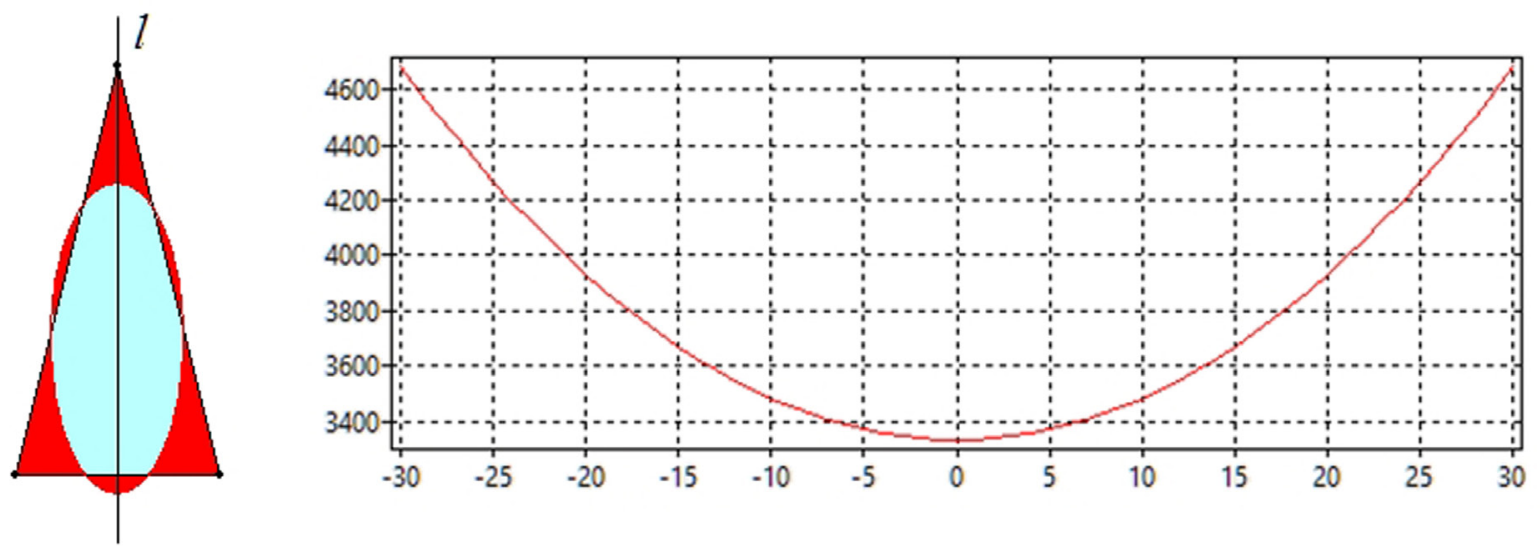

Рис. 3. Равнобедренный треугольник с наложенным аппроксимирующим эллипсом (слева, красным выделена суммарная площадь их непересекающихся частей S) и график зависимости S от смещения центра эллипса от барищентра треугольника вдоль прямой l (справа, положительное направление смещения - вверх). 


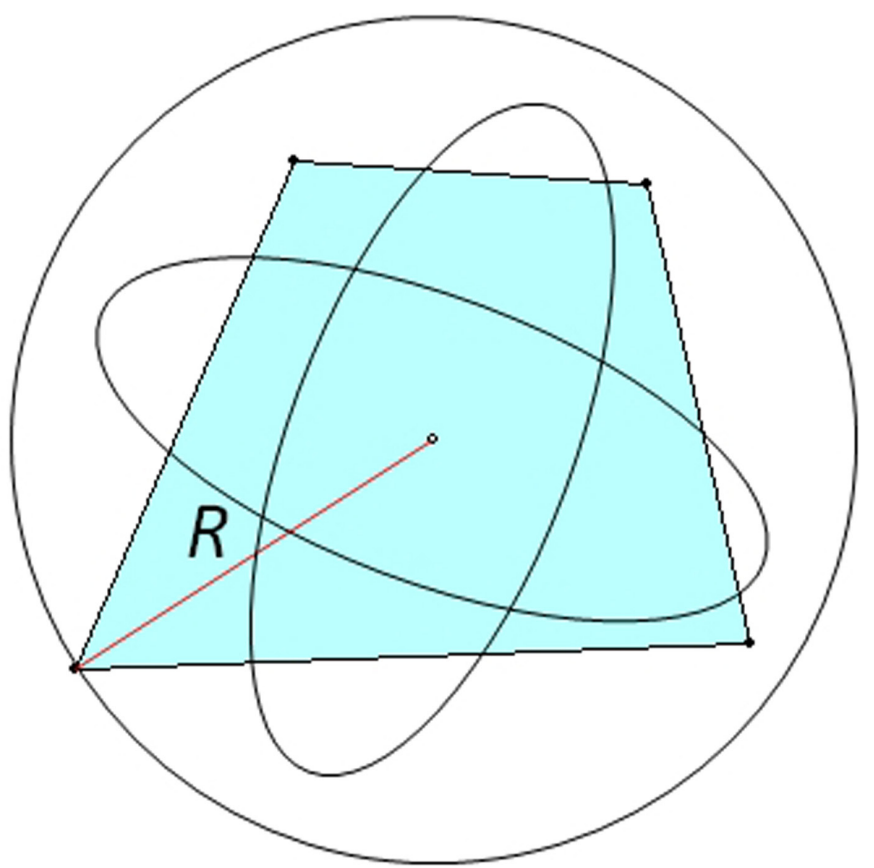

Рис. 4. Четырёхугольник внутри окружности с иентром в его барицентре и радиусом $R$ и два взаимно перпендикулярных эллипса с полуосями а и $b$ или, что то же самое, два одинаково ориентированных эллипса с полуосями $a, b u b, a$ coответственно.

ческий интерес с точки зрения их строгих доказательств или опровержений. Здесь же мы ограничимся рядом примеров.

Рассмотрим равнобедренный треугольник с длиной основания 100 ед. и высотой 200 (рис. 3). Из соображений симметрии и удлинения треугольника, большая полуось аппроксимирующего эллипса будет лежать на одной прямой $l$ с высотой треугольника. Это позволяет варьировать только одну координату центра эллипса. На графике видно, что величина $S$ минимальна при нулевом смещении центра эллипса от барицентра треугольника вдоль прямой $l$ (симметричность графика является побочным эффектом).

На рис. 4 изображён четырёхугольник, вокруг которого проведена окружность с центром в барицентре четырёхугольника и радиусом $R$, равным расстоянию от барицентра до наиболее удалённой от него вершины четырёхугольника. Меньше $R$ быть не может, так как является оптимальным для эллипса с $a=b=R$, аппроксимирующего правильный многоугольник с бесконечным числом вершин и дающего минимально возможную нулевую $S$. Большее значение $R$ приводит только к увеличению $S$. Рассмотрим множество аппроксимирующих эллипсов, одинаково ориентированных относительно многоугольника, и пусть длины их полуосей независимо изменяются от 0 до R. Тогда для любого эллипса с полуосями $a$ и $b$ всегда найдётся эллипс с полуосями $b$ и $a$, совпадающий по форме с исходным, но повёрнутый относительно него на $90^{\circ}$ (внутренние эллипсы на рис. 4). Оба этих эллипса в силу симметрии совпадают с самими собой, повёрнутыми на $180^{\circ}$. В итоге, перебор всех возможных ориентировок эллипсов в пределах прямого угла оказывается исчерпывающим, так как при независимом переборе 

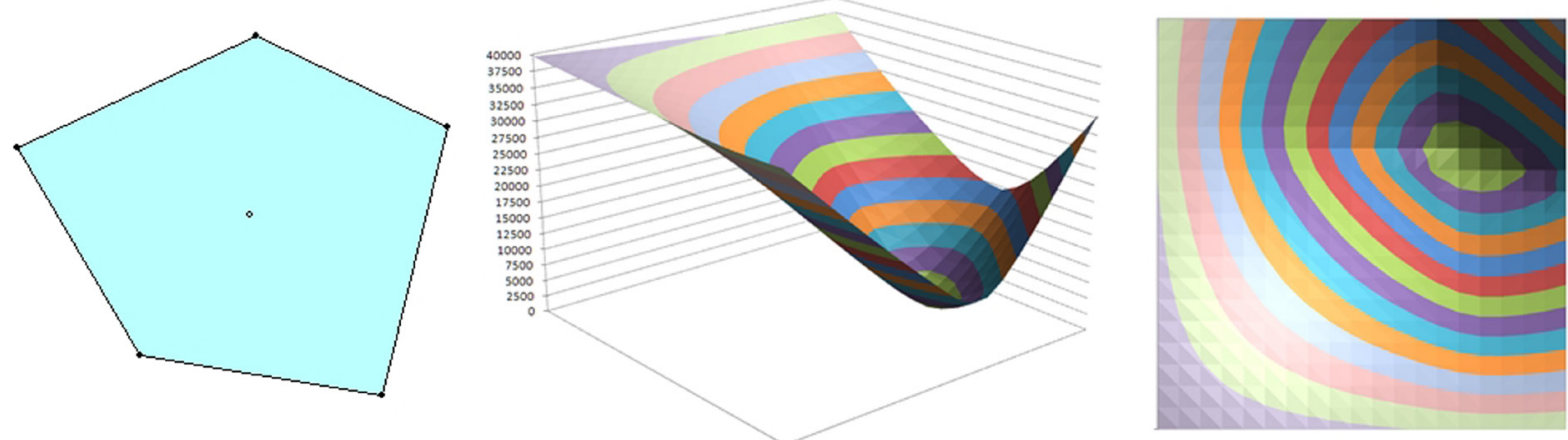

Рис. 5. Пятиугольник и график функции $S(a, b)$ в перспективе и в плане.

длин полуосей, каждый эллипс, как бы проходит четыре позиции, отличающиеся друг от друга поворотом на прямой угол.

Для пятиугольника на рис. 5. тут же приведён график функции $S(a, b)$ при вертикальной ориентировке аппроксимирующего эллипса. Видно, что график представляет собой непрерывную поверхность с единственным минимумом в зелёной области.

Наконец, рис. 6. иллюстрирует характер зависимости $S_{\min }(\alpha)$ от ориентировки аппроксимирующего эллипса на примере шестиугольника. Единственный минимум функции достигается при повороте большой оси аппроксимирующего эллипса примерно на $25^{\circ}$ относительно горизонтальной оси координат.

Указанный подход был реализован автором в свободно распространяемой IDE Lazarus (версия 1.6.4) на языке Object Pascal в виде приложения, позволяющего по введённым координатам вершин многоугольника вычислять положение и форму аппроксимирующего эллипса. В качестве числовой характеристики облика многоугольника было взято отношение $f$ длин меньшей полуоси к большей. Примем, что многогранник имеет вытянутый (или, что то же самое для плоского случая, уплощённый) облик, если $f<0.5$ и изометричный облик, если $f>0.5$. Само $f$, очевидно, изменяется в пределах от 0 до 1. На рис. 7 показаны примеры работы приложения - ряд многоугольников и аппроксимирующих их эллипсов, с указанием направления большой оси эллипсов и отношения $f$. Отметим, что правильные многоугольники (например, пятиугольник на рис. 7) аппроксимируются окружностями (эллип-
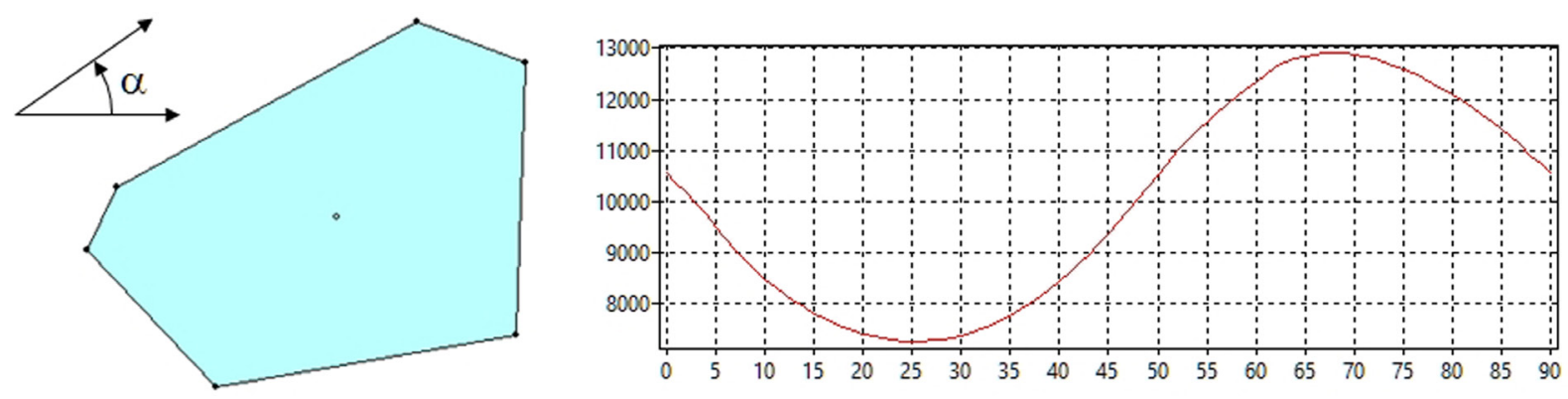

Рис. 6. Шестиугольник и график функиии $S_{\text {min }}(\alpha)$. 
сами с равными длинами полуосей), а прямоугольники (например, прямоугольник на рис. 7) - эллипсами, для которых $f$ совпадает с отношением сторон прямоугольника. Среди всех указанных на рис. 7 многоугольников вытянутым можно считать только последний с $f=0.19$. Прямоугольник с $f=0.5$ в равной степени может считаться как вытянутым, так и изометричным.

Полученные пилотные результаты подтвердили эффективность предлагаемого способа оценки облика двумерного кристалла, что даёт основания для его расширения на трёхмерный случай - для оценки облика реальных искажённых кристаллов.

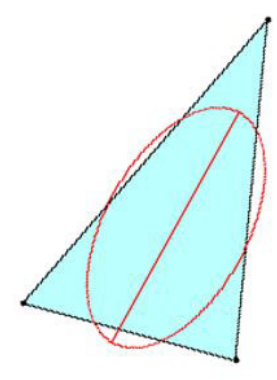

0.55

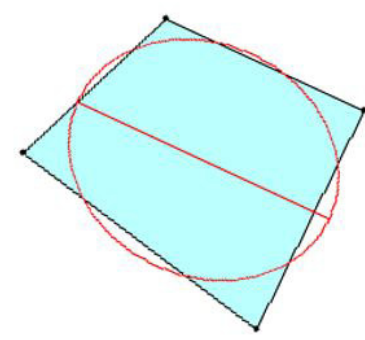

0.84

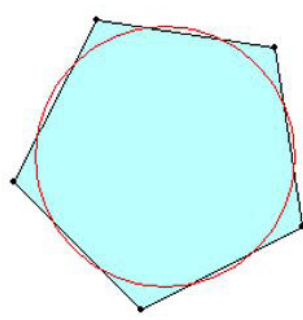

1

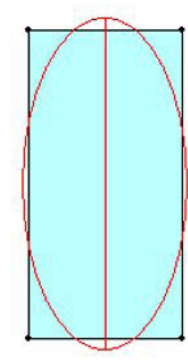

0.5

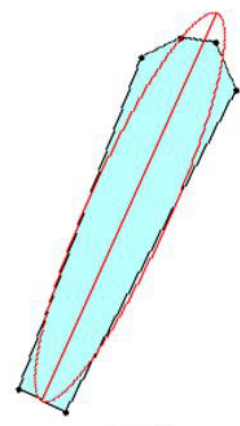

0.19

Рис. 7. Примеры многоугольников и аппроксимирующих их эллипсов. Снизу приведень отношения длин меньшей полуоси к большей.

\section{Список литературы}

1. Степенщиков Д.Г., Войтеховский Ю.Л. О связи облика и реальной формы кристалла // Вестник КНЦ РАН. № 1. 2012. С. 145-148.

2. Степенщиков Д.Г. Облик как характеристика искажённых кристаллов // Материалы юбилейного съезда Российского минералогического общества «200 лет РМО». СПб. 2017. Т.1. С. 132-133.

3. Степенщиков Д.Г. Аппроксимация облика кристалла трёхосным эллипсоидом // Тр. Ферсмановской науч. сессии ГИ КНЦ РАН. 2018. 15. С. 357-360. 4. Шафрановский И.И. О кристалломорфологическом описании микрокристаллических минералов // Минералогический сборник Львовского геологического общества. 1959. № 13. С. 73-83. 